Case Report

\title{
Clinical Case with Negative Polymerase Chain Reaction (PCR) and Suspicious Chest Computed Tomography (CT) Images SARS-CoV-2 Infection or Not
}

\author{
Adjagba Philippe Mahouna ${ }^{1, ~}$, Adjadohoun Sonia ${ }^{3}$, Hounkponou Murielle ${ }^{1}$, Sonou Arnaud ${ }^{1}$, \\ Codjo Léopold $^{5}$, Soho Edson ${ }^{4}$, Dahito Mickaella ${ }^{1}$, Avocèvou Géraud ${ }^{1}$, Djoh Ingrid ${ }^{1}$, \\ Sissinto Yolande ${ }^{2}$, Yèkpè Patricia ${ }^{3}$, Biaou Olivier ${ }^{3}$, Houenassi Dèdonougbo Martin ${ }^{1}$ \\ ${ }^{1}$ University Clinic of Cardiology, CNHU-HKM, University of Abomey-Calavi (UAC), Cotonou, Benin \\ ${ }^{2}$ University Clinic of Microbiology and Parasitology, CNHU-HKM, University of Abomey-Calavi (UAC), Cotonou, Benin \\ ${ }^{3}$ University Clinic of Imaging and Radiodiagnostic, CNHU-HKM, University of Abomey-Calavi (UAC), Cotonou, Benin \\ ${ }^{4}$ Radiography, Ultrasound and Scanning Centre, Cotonou, Benin \\ ${ }^{5}$ University Clinic of Cardiology, CHUDBA, University of Parakou, Parakou, Benin
}

Email address:

dotoup $@$ yahoo.fr (A. P. Mahouna)

${ }^{*}$ Corresponding author

\section{To cite this article:}

Adjagba Philippe Mahouna, Adjadohoun Sonia, Hounkponou Murielle, Sonou Arnaud, Codjo Léopold, Soho Edson, Dahito Mickaella, Avocèvou Géraud, Djoh Ingrid, Sissinto Yolande, Yèkpè Patricia, Biaou Olivier, Houenassi Dèdonougbo Martin. Clinical Case with Negative Polymerase Chain Reaction (PCR) and Suspicious Chest Computed Tomography (CT) Images SARS-CoV-2 Infection or Not. American Journal of Internal Medicine. Vol. 8, No. 4, 2020, pp. 148-152. doi: 10.11648/j.ajim.20200804.12

Received: May 12, 2020; Accepted: May 28, 2020; Published: June 4, 2020

\begin{abstract}
Background: The SARS-CoV-2 infection has polymorphic clinical presentations. The real time PCR is the reference diagnostic test; however, it can only detect presence of virus for a specific window of time. Case: We report a clinical case in a patient aged 66 years. His clinical history included known hypertension for 10 years and ischemic stroke. He had no known contact with infected persons. He initially presented with a productive cough, fever, shortness of breath on exertion, intense asthenia and palpitations. The real time PCR with upper airway samples was conducted on days 18 and 20 of the onset of symptoms and was negative. Despite chest CT abnormalities, the patient was not considered to be infected SARS-CoV-2 according to national recommendations for diagnosis and treatment in Benin. He was discharged from the treatment centre. Readmitted 7 days later to the emergency room for respiratory distress, the patient died. Conclusion. Diagnosis of SARS-CoV-2 infection can be difficult. In the context of typical clinical presentation, chest CT features of viral pneumonia may be strongly suspicious for SARS-CoV-2 despite negative real time PCR results. In order to improve the diagnostic and therapeutic strategy for SARS-CoV-2 infection in Benin, chest CT and other diagnostic tests/ criteria should be adopted.
\end{abstract}

Keywords: SARS-CoV-2, Real Time PCR, False Negative, Chest CT

\section{Introduction}

On 31 December 2019, China notified WHO about a cluster of pneumonia cases of unknown causes in Wuhan, Hubei province. The causative pathogen, was later identified and named as severe acute respiratory syndrome coronavirus 2 (SARS-CoV-2) by the Coronavirus Study Group of the
International Committee on Taxonomy of Viruses based on its phylogeny, taxonomy, and established practices $[1,2]$ (Since then, the SARS-CoV-2 has spread worldwide and was declared a pandemic by the WHO on March 11, 2020 [3]. Africa is one of the last continents to be affected by SARS-CoV-2 with relatively few reported cases and deaths. In Benin, where 210 cases have been reported [4], the national reporting and treatment strategy remains based on 
confirmation of cases by real time PCR. Several data in the literature report false negative results by real time PCR [5-9]. In these cases, the use of other diagnostic tools such as chest Computed Tomography (CT) and serological tests is necessary. Chest CT has proven to be a credible alternative diagnosis in patients with typical images $[6,7,10]$. We report a clinical case in a patient with typical chest CT scan images with two negative real time PCR test. The analysis of the clinical, chest CT and evolutionary data of this patient may change the management and prevention of the infection in our country.

\section{Case}

The patient was a male, aged 66 years, weighing $82 \mathrm{kgs}$ for a height of $187 \mathrm{~cm}$. He lived $133 \mathrm{~km}$ from the city of Cotonou, where the highest number of cases of SARS-CoV-2 infection have been recorded in Benin. His clinical history included known hypertension for 10 years and ischemic stroke. He had no known contact with anyone infected with SARS-CoV-2 and no cases had been identified in his city of residence at the time of his symptoms. He initially presented with a productive cough, fever of $38.5^{\circ} \mathrm{C}$, then secondarily with shortness of breath on exertion, intense asthenia and palpitations. He was admitted 5 days later, for intensive care in his hometown hospital and treated for pulmonary embolism associated with pneumonia with enoxaparin (8,000 UI twice a day) and antibiotics (Roxithromycin $150 \mathrm{mg}+$ Amoxycillin/Clavulanic acid $1 \mathrm{~g}$, twice a day).

After a 5-day stay in intensive care with clinical stabilization, he was transferred under rivaroxaban $(15 \mathrm{mg}$ twice a day) to Cotonou for CT scan and was admitted to the cardiology department of the "'Centre National Hospitalier et Universitaire Hubert Koutoukou Maga', (CNHU-HKM). He presented a sudden deterioration of his clinical condition on the day of admission with respiratory distress $\mathrm{SpO} 2$ of $80 \%$ and a fever of $40^{\circ} \mathrm{C}$. He was given non-invasive respiratory assistance with high concentration oxygen by mask. The patient's biological data are summarized in Table 1. The ECG showed no particularities other than bi-atrial overload and isolated ventricular extrasystole. The echocardiographic findings were signs of an acute pulmonary heart with RV/LV ratio at 0.87 , pulmonary artery systolic pressure (PASP) using tricuspid valve velocity at $50 \mathrm{mmHg}$. Kinetics and function of the left ventricle were good. There was no significant mitro-aortic valve disease or pericardial effusion.

Table 1. Biological data.

\begin{tabular}{ll}
\hline & Patient values (ULN) \\
\hline D-dimer (ng/l) & 10,000 (20X ULN) \\
Complete blood count (CBC) & 15.7 \\
Hemoglobin (grams/dl) & 305 \\
Platelets (billion/l) & 18,7 \\
White blood cells (billion/l) & 91 \\
Neutrophils (\%) & 4 \\
Lymphocytes (\%) & \\
\hline
\end{tabular}

\begin{tabular}{ll}
\hline & Patient values (ULN) \\
\hline Protonin Ic (ng/l) & $37(2.6 \mathrm{X} \mathrm{ULN})$ \\
Blood urea nitrogen (BUN) (g/l) & 0.17 \\
Creatinine (mg/l) & 11.5 \\
C-Reactive Protein (CRP) (mg/l) & $82.3(5.6 \mathrm{X} \mathrm{ULN})$ \\
real time PCR (test1) & negative \\
real time PCR (test2) & negative \\
\hline
\end{tabular}

ULN: Upper Limit Normal; PCR: Polymerase Chain Reaction.

The chest CT scan showed the following abnormalities: Frosted glass lung areas bilateral asymetrical predominantly posterior lung more pronounced on the right, of mixed topography, diffuse condensation on the right with lower lobe aerial bronchogram (figure 1 and figure 3), ground-glass opacity, central basal posterior left, focal lung infarction (figure 2), lower lobar pulmonary embolism and sub-segmental and segmental (figures 3 and figure 4), dilatation of right cavities, low-abundant pleural fluid effusion on the right (figure 3).

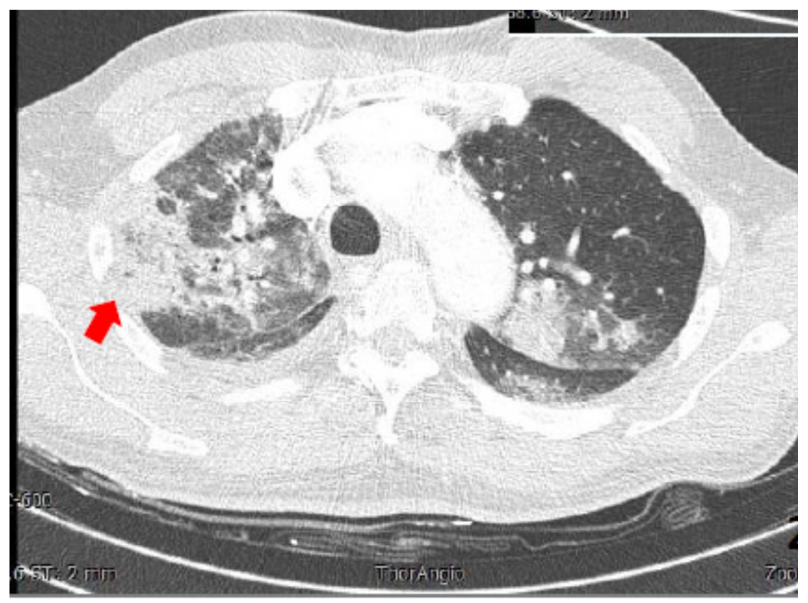

Figure 1. Chest CT imaging.

Frosted glass lung areas bilateral asymetrical predominantly posterior more pronounced on the right (red arrows).

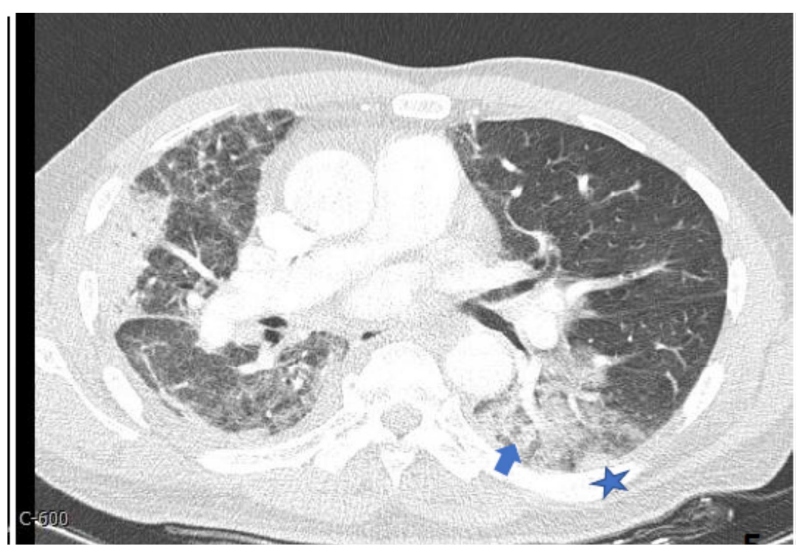

Figure 2. Chest CT imaging.

Ground-glass opacity, central basal posterior left (blue arrow), Focal Lung Infarction (star). 


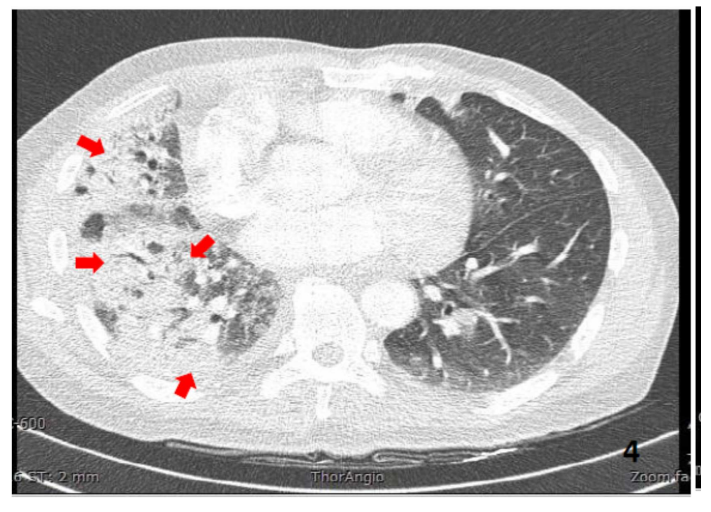

Figure 3. Chest CT imaging.

Diffuse lung condensation on the right with lower lobe aerial bronchogram (red arrows).

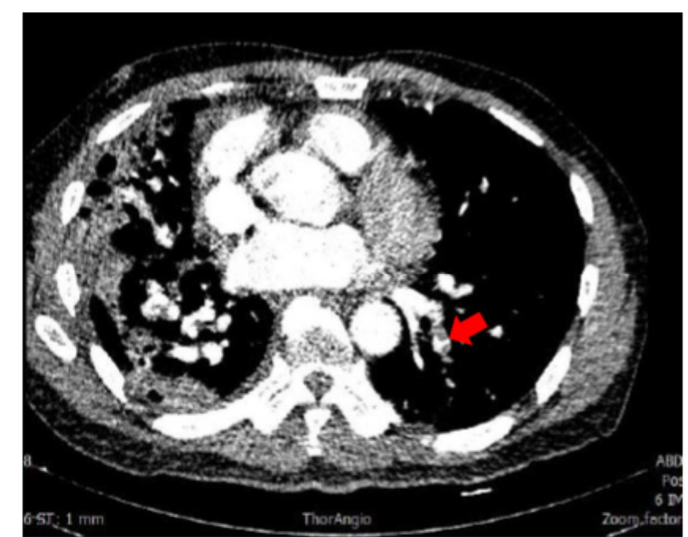

Cardiovascular abnormalities: Left segmental embolism (red arrows).

Figure 4. Chest CT imaging.

Based on the chest CT scan results a SARS-CoV-2 infection was suspected, and the patient was transferred to the national SARS-CoV-2 management center in accordance with national policy. The disinfection procedures of the room and of the equipment were made and the 4 contacts patients put isolation as recommended.

Confirmatory testing by real time PCR with oropharyngeal samples was performed on days 18 and 20 of the onset of symptoms at the national SARS-CoV-2 management center. real time PCR results were negative twice. The patient was not considered to be infected with SARS-CoV-2 and was not treated as such. He was discharged from the treatment centre and returned home. Readmitted to the emergency room for respiratory distress 7 days later, the patient died.

\section{Discussion}

This clinical case in a patient with a history of hypertension and cerebrovascular disease highlights several points for comment, including:

1. the patient's clinical symptomatology and medical history,

2. the diagnosis of SARS-CoV-2 infection,

3 . the therapeutic regimen and hospital management of the patient and the possible consequences of a falsely negative result for SARS-CoV-2.

\subsection{The Patient's Clinical Symptomatology and Medical History}

The patient presented with respiratory symptoms and fever suggestive of pulmonary embolism and/or pneumonia. Pulmonary embolism is one of the common clinical presentations or complications of SARS-CoV-2 infection as reported in several data in the literature [11-15]. SARS-CoV-2 is associated with a high thromboembolic risk, with several factors being incriminated: prolonged immobilization, inflammation responsible for a state of hypercoagulability and endothelial dysfunction. High levels of D-dimer $(>1 \mathrm{~g} / \mathrm{L})$ were strongly correlated with high intra-hospital mortality [16] or 6 times the upper limit of normal [17]. The patient described in the clinical case had a D-dimer level of 20 times the upper limit of normal.

The patient was 66 years old, had hypertension and stroke in his history. Elderly patients with a cardiovascular history appear to be more affected with a poor prognosis than in the absence of cardiovascular disease as reported in the literature. Indeed, SARS-CoV-2 has a dual effect at the cardiovascular level: the infection will be more intense if the host has cardiovascular comorbidities and the virus itself can cause potentially fatal cardiovascular damage [18]. In a study of 138 cases of SARS-CoV-2 infection in the Wuhan region of China, 64 patients $(46.4 \%)$ had at least one predominantly CV comorbidity, hypertension was present in $31.2 \%$ of subjects, diabetes in $10.1 \%$ of subjects and cardiovascular infection in $14.5 \%$ of patients [19]. In this study, the forms of SARS-CoV-2 that stayed in the Intensive Care Unit (ICU) were higher in patients with hypertension $(50.3 \%)$, diabetes $(22.2 \%)$, heart disease $(25 \%)$ and cerebrovascular disease (16.7\%) [19]. Higher excess mortality occurred in the group of patients with a cardiovascular history $(10.5 \%)$ compared to patients with underlying chronic respiratory disease $(6.3 \%)$.

\subsection{Diagnosis of SARS-CoV-2 Infection in the Patient}

The confirmation of the diagnosis of SARS-CoV-2 infection was not made by the real time PCR test, which was negative twice. Several data in the literature report false negative results by real time PCR [5-9]. A recent study showed that the mean duration of viral clearance in patients with SARS-CoV-2 in China was 20 days [20]. The real time PCR is the reference test because it is very specific. However, this technique lacks sensitivity. False-negative results are related to low viral load, the quality and timing of sampling (e.g. during the early phase of infection and then during the symptom resolution phase), the presence of PCR inhibitors, virus mutation and transport conditions [21]. When the test returns negative, it must be repeated and at different sites. The study by Wang et al. compared the performance of real time PCR viral RNA testing at different collection sites on 1070 samples obtained from 205 patients with confirmed SARS-CoV-2 based on a combination of compatible symptoms and characteristic radiological signs. The most 
sensitive specimen appeared to be bronchoalveolar lavage $(93 \%)$, followed by sputum $(72 \%)$. The widely used nasal swab real time PCR appeared to be less sensitive $(63 \%)$ and should be repeated to reduce the incidence of false negatives [22]. In the clinical case patient, the swab was taken from the oropharynx.

It was the chest $\mathrm{CT}$ images that made the diagnosis by finding two characteristic anomalies: ground-glass opacity with peripheral distribution and condensation. The pulmonary embolism found may be an evolving complication. Indeed, pulmonary embolism is on the left while lesions of the pulmonary parenchyma predominate on the right.

In these cases, chest CT represents an alternative in symptomatic patients by showing typical lesions $[6,7,10]$. Ai T. et al. showed a sensitivity of $97 \%$ chest CT scan, based on positive real time PCR results. These same authors had 75\% chest CT positive cases in real time PCR negative patients [23]. The specificity of the CT scan seems more modest, however a recent Chinese American study [24] has shown the possibility of distinguishing SARS-CoV-2 infection from other viral pneumopathies. The most discriminating features for SARS-CoV-2 pneumonia included a peripheral distribution ( $80 \%$ vs. $57 \%$, p $<0.001$ ), ground-glass opacity ( $91 \%$ vs. $68 \%$, $\mathrm{p}<0.001)$ and vascular thickening (58\% vs. $22 \%, \mathrm{p}<0.001)$.

Serological tests can also be performed if real time PCR is negative with a strong suspicion of SARS-CoV-2 infection. They detect antibodies specific for SARS-CoV-2 infection of IgM and IgG type [21]. These tests are not true diagnostic tests. The antibodies are not produced at the onset of infection, but somewhat later (from day 5 and day 10 of the onset of symptoms for IgM and IgG respectively) and thus one can miss out on newly infected cases. Furthermore, these tests do not tell whether a person is still infectious, unlike real time PCR tests, which also reveal the virus load in the sample. A positive result from these tests, however, proves contact with the virus, regardless of whether symptoms are present or not. Serological tests would have been of interest in this patient but were not available in the SARS-CoV-2 diagnostic system in Benin.

\subsection{The Therapeutic Regimen and Hospital Management of the Patient and the Possible Consequences of a Falsely Negative Result for SARS-CoV-2}

The patient in the clinical case was not considered to be infected with SARS-CoV-2 according to national recommendations for diagnosis and management despite compatible chest CT images. The management of this patient revealed gaps in the case management policy and failure to consider CT abnormalities in the definition of suspected SARS-CoV-2 infection. Several consequences can be noted for a patient falsely declared not infected with SARS-CoV-2 infection. Individuals with these results may relax physical distancing and other personal measures designed to reduce the transmission of the virus to others. In the case of clinicians, they may be sent to the frontlines of care and inadvertently transmit the virus to patients and colleagues, further straining the already precarious ability of the health care system to respond to the pandemic [9].

\section{Conclusion}

SARS-CoV-2 infection with its polymorphic clinical presentations can be difficult to diagnose. In the context of typical clinical presentation, chest CT features of viral pneumonia may be strongly suspicious for SARS-CoV-2 despite negative real time PCR results. In order to improve the diagnostic and therapeutic strategy for SARS-CoV-2 infection in Benin, chest CT and other diagnostic tests / criteria should be adopted.

\section{References}

[1] Gorbalenya AE, Baker SC, Baric RS, de Groot RJ, Drosten C, Gulyaeva AA, et al. Severe acute respiratory syndrome-related coronavirus: the species and its viruses - a statement of the Coronavirus Study Group. bioRxiv, 2020. Available at: https:// doi.org/10.1101/2020.02.07.937862. Accessed February 11, 2020 .

[2] Zhu N, Zhang D, Wang W, Li X, Yang B, Song J, et al.; China Novel Coronavirus Investigating and Research Team. A novel Coronavirus from patients with pneumonia in China, 2019. N Engl J Med 2020; 382: 727-33.

[3] OMS. COVID-19 - Chronologie de l'action de l'OMS. https://www.who.int/fr/news-room/detail/08-04-2020-who-tim eline---covid-19. Accessed April 17, 2020.

[4] Coronavirus (Covid-19). Tout savoir sur la gestion de la pandémie du coronavirus au Bénin. https://www.gouv.bj/coronavirus/. Accessed May 27, 2020.

[5] Xiao AT, Tong YX, Zhang S. False-negative of RT-PCR and prolonged nucleic acid conversion in COVID-19: Rather than recurrence. J Med Virol. 2020. doi: 10.1002/jmv.25855.

[6] Li D, Wang D, Dong J, Wang N, Huang H, Xu H, Xia C. False-Negative Results of Real-Time Reverse-Transcriptase Polymerase Chain Reaction for Severe Acute Respiratory Syndrome Coronavirus 2: Role of Deep-Learning-Based CT Diagnosis and Insights from Two Cases. Korean J Radiol. 2020; 21 (4): 505-508. doi: 10.3348/kjr.2020.0146.

[7] Feng H, Liu Y, Lv M, Zhong J. A case report of COVID-19 with false negative RT-PCR test: necessity of chest CT. Jpn J Radiol. 2020. doi: 10.1007/s11604-020-00967-9.

[8] Pan Y, Long L, Zhang D, Yan T, Cui S, Yang P, Wang Q, Ren S. Potential false-negative nucleic acid testing results for Severe Acute Respiratory Syndrome Coronavirus 2 from thermal inactivation of samples with low viral loads. Clin Chem. 2020 Apr 4: hvaa091. doi: 10.1093/clinchem/hvaa091.

[9] Colin P. West, Victor M. Montori, Priya Sampathkumar. COVID-19 Testing: The Threat of False-Negative Results. Mayo Clinic Proceedings, 2020; DOI: 10.1016/j.mayocp.2020.04.004.

[10] Chunqin Long, Huaxiang Xu, Qinglin Shen, Xianghai Zhang, Bing Fan, Chuanhong Wang, Bingliang Zeng, Zicong Li, Xiaofen Li, Honglu Li. Diagnosis of the Coronavirus Disease (COVID-19): rRT-PCR or CT? Eur J Radiol. 2020 May; 126: 108961. doi: 10.1016/j.ejrad.2020.108961. 
[11] Yuanliang Xie, Xiang Wang, Pei Yang, Shutong Zhang. COVID-19 Complicated by Acute Pulmonary Embolism. Radiology: Cardiothoracic Imaging 2020; 2 (2): e200067 https://doi.org/10.1148/ryct.2020200067.

[12] Grillet F, Behr J, Calme P, Aubry S, Delabrousse E. Acute Pulmonary Embolism Associated with COVID-19 Pneumonia detected by pulmonary CT angiography. Radiology. $2020 \mathrm{Apr}$ 23; 201544. doi: 10.1148/radiol.2020201544. Online ahead of print.

[13] Chen N, Zhou M, Dong X, et al. Epidemiological and clinical characteristics of 99 cases of 2019 novel coronavirus pneumonia in Wuhan, China: a descriptive study. Lancet. 2020; 395 (10223): 507-513.

[14] Huang C, Wang Y, Li X, et al. Clinical features of patients infected with 2019 novel coronavirus in Wuhan, China. Lancet. 2020; 395 (10223): 497-506.

[15] Tang N, Li D, Wang X, Sun Z. Abnormal Coagulation parameters are associated with poor prognosis in patients with novel coronavirus pneumonia. J Thromb Haemost. 2020 [published online ahead of print].

[16] Zhou F, Yu T, Du R, et al. Clinical course and risk factors for mortality of adult inpatients with COVID-19 in Wuhan, China: a retrospective cohort study. Lancet. 2020 Mar 28; 395 (10229): 1054-1062. doi: 10.1016/S0140-6736(20)30566-3.

[17] Tang N, Bai H, Chen X, Gong J, Li D, Sun Z. Anticoagulant treatment is associated with decreased mortality in severe coronavirus disease 2019 patients with coagulopathy. J Thromb Haemost. 2020 May; 18 (5): 1094-1099. doi: $10.1111 /$ jth.14817.

[18] El Boussadani B, Benajiba C, Aajal A, Ait Brik A, Ammour O, El Hangouch J, Oussama O, Oussama B, Tahiri N, Raissuni Z.
Pandémie COVID-19: impact sur le systeme cardiovasculaire. Données disponibles au $1^{\text {er }}$ avril 2020. Ann Cardiol Angeiol (Paris). 2020 Apr 7; S0003-3928(20)30056-1. doi: 10.1016/j.ancard.2020.04.001.

[19] Wang D, Hu B, Hu C, et al. Clinical Characteristics of 138 Hospitalized Patients With 2019 Novel Coronavirus-Infected Pneumonia in Wuhan, China. JAMA. 2020 Feb 7; 323 (11): 1061-1069. doi: 10.1001/jama.2020.1585.

[20] Zhou F, Yu T, Du R, Fan G, Liu Y, Liu Z, et al. Clinical course and risk factors for mortality of adult inpatients with COVID-19 in Wuhan, China: a retrospective cohort study. Lancet. 2020 Mar 11. pii: S0140-6736(20)30566-3. doi: 10.1016/S0140-6736(20)30566-3.

[21] Thabet L, Mhalla S, Hannachi N, Karray Hakim H, Trabelsi A, Dhaouadi H, Msselmani S. Stratégie du Diagnostic virologique $\mathrm{du} \quad$ SARS-CoV-2 https://www.macsievents.com/stbc/documents/b6ee4a1f3c9d8 f535db7ac3d0ae175e9.pdf. Consulté le 24 Mars 2020.

[22] Wang W, Xu Y, Gao R, Lu R, Han K, Wu G, et al. Detection of SARS-CoV-2 in Different Types of Clinical Specimens. JAMA. 2020 Mar 11; e203786. doi: 10.1001/jama.2020.3786.

[23] Tao Ai, Zhenlu Yang, Hongyan Hou, Chenao Zhan, Chong Chen, Wenzhi Lv, Qian Tao, Ziyong Sun, Liming Xia. Correlation of Chest CT and RT-PCR Testing in Coronavirus Disease 2019 (COVID-19) in China: A Report of 1014 Cases. Radiology. 2020; 200642. doi: 10.1148/radiol.2020200642.

[24] Harrison X Bai, Ben Hsieh, Zeng Xiong, Kasey Halsey, Ji Whae Choi, Thi My Linh Tran, et al. Performance of radiologists in differentiating COVID-19 from viral pneumonia on chest CT. Radiology. 2020 Mar 10; 200823. doi: 10.1148/radiol.2020200823. 